\title{
Dislocación testicular postraumática
}

\author{
Luján Marco S, Budía Alba A, Bango García V, Ramirez Backhaus M, Delgado Oliva FJ, \\ Jimenez Cruz JF.
}

Servicio de Urología. Hospital Universitario La Fe. Valencia.

Actas Urol Esp 2006; 30 (4): 409-411

\section{RESUMEN \\ DISLOCACIÓN TESTICULAR POSTRAUMÁTICA}

Presentamos el caso clínico de un paciente politraumatizado, conductor de motocicleta, con dislocación testicular derecha. Se trata de una lesión infrecuente tras traumatismo contuso que requiere diagnóstico y tratamiento precoz con el fin de evitar la atrofia testicular. Esta lesión deberá tenerse en cuenta en toda evaluación del politraumatizado, requiriendo tratamiento quirúrgico. El objetivo del caso clínico es aportar un nuevo caso a la literatura, siendo este el sexto caso publicado por autores españoles, con el fin de clarificar el manejo y tratamiento.

Palabras claves: Luxación testicular. Dislocación testicular.

\section{ABSTRACT}

TRAUMATIC TESTICULAR DISLOCATION

Testicular dislocation is an uncommon injury after blunt scrotal trauma (with only 150 cases reported) that must be properly diagnosed and treat in order to avoid a testicular atrophy. We presented a case report of traumatic dislocation of right testicle after a motorcycle accident. This type of testicular injury should be included in the whole evaluation of every polytrauma patient and usually requires a surgical treatment. The aim of this report is to clarify some aspects of the management of such rare entity.

Keywords: Testicular luxation. Testicular dislocation.

$\mathrm{L}$ a dislocación o luxación es un traumatismo testicular no penetrante de escasa frecuencia, con pocos casos publicados en literatura. El tratamiento consiste en la reducción del testículo luxado. Aportamos el caso clínico con dicha lesión, analizando el proceso diagnóstico y terapéutico.

\section{CASO CLÍnico}

Paciente de 31 años de edad que tras accidente de tráfico con motocicleta es trasladado al servicio de urgencias de nuestro hospital. Presentaba lesiones faciales, fracturas óseas múltiples, contusión pulmonar y tumoración inguinal derecha. A la exploración clínica se apreció herida inciso-contusa de bordes bien definidos de la porción dorsal peneana de unos $5 \mathrm{~cm}$ de longitud afectando exclusivamente a piel, sin herida alguna sobre bolsa escrotal. Se evidencia el signo de Brockman (bolsa escrotal vacía y bien desarrollada) con testículo izquierdo normal y teste derecho alojado en canal inguinal ipsilateral doloroso al tacto $\mathrm{y}$ con imposibilidad de descenso manual. El paciente no refiere antecedentes de anomalías testiculares.

Estando hemodinámicamente estable, se solicito análisis sanguíneo sin hallazgos anormales y pruebas de imagen (TAC craneal-torácico-abomino-pélvico) donde no se evidenció lesión de órganos intraabdominales, pero si contusión pulmonar bilateral con infiltrados alveolares en segmento anterior de ambos lóbulos superiores. Se 
apreció estructura de morfología ovoidea en canal inguinal derecho, correspondiente al testículo luxado (Fig. 1). Asímismo, presentaba fractura radio-cubital izquierda, fractura de clavícula y fractura de $4^{\circ}-5^{\circ}$ metatarsiano de mano derecha.

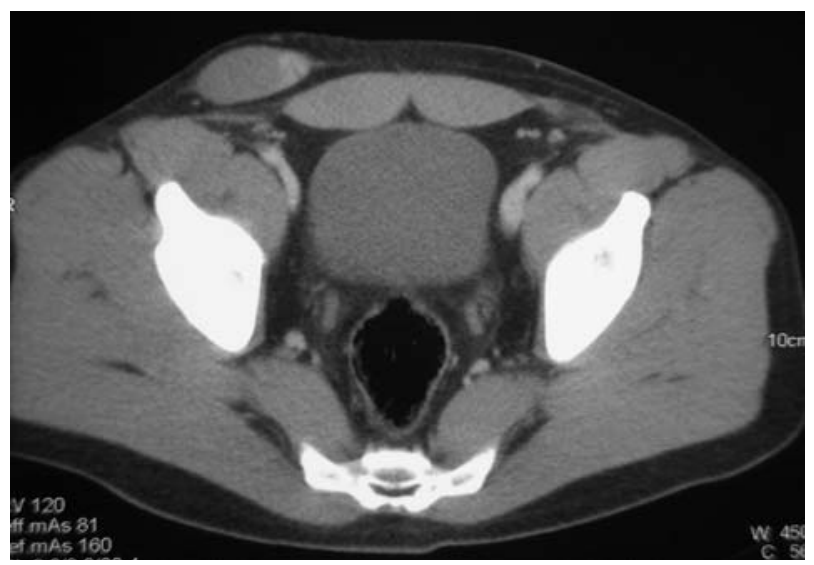

FIGURA 1. Imagen de TAC en corte abdominal con imagen ovoidea sobre pared abdominal correspondiente al testiculo derecho dislocado.

Con el diagnóstico de traumatismo testicular derecho y luxación del mismo, se decidió intervención quirúrgica urgente debido a imposibilidad de reducción manual. Con los relajantes musculares administrados durante el acto anestésico se consiguió descenso manual del testículo a la bolsa escrotal, pero con dislocación espontánea tras su reposición, por lo que procedimos a orquidopexia. No se apreció lesión en parénquima testicular, tan solo pequeño hematoma en la albuginea en dicho testículo. Finalmente se realizó cierre con puntos sueltos de la lesión peneana.

El paciente es ingresado en el servicio de traumatología tras la intervención, con postoperatorio sin complicaciones y visitado posteriormente en nuestras consultas externas, procediéndose al alta hospitalaria tras controles clínicos y ecográficos que mostraron testículos intraescrotales con una morfología normal.

\section{DISCUSIÓN}

Nos encontramos ante una entidad clinica poco frecuente, publicada por primera vez en 1818 por Claurby, con 150 casos referenciados en la literatura, de los cuales 5 publicados por autores españoles y uno por autores de Guatemala ${ }^{1-6}$. Algunos de estos establecen que la dislocación está en función de la fuerza traumática, de tal forma que si es menor de $50 \mathrm{Kg}$. se produce una dislocación, mientras que si es mayor existirá una rotura de la albugínea al sobrepasar la resistencia tisular $^{1}$.

La mayoría de las dislocaciones son unilaterales (90\%), superficiales (80\%), y de localización inguinal superficial (50\% casos). La lesión es producida básicamente por un traumatismo, siendo más frecuente en los accidentes de moto, por contusión directa contra el depósito de gasolina o sobre el manillar ${ }^{7}$. Otros mecanismos de producción menos frecuentes son: contusión directa sobre la bolsa escrotal, practicas deportivas como fútbol, ciclismo, etc. Lo más frecuente es que la bolsa escrotal contacte con el pubis y evite la luxación, pero si esto no se produce, el testículo en sus maniobras de ascenso rompe fibras musculares del cordón espermático y crea un "hojal" que impide la vuelta a su posición inicial colocándose entre la fascia del músculo oblicuo mayor y tejido celular subcutáneo ${ }^{8}$. Se ha descrito que anomalías tales como la hernia inguinal indirecta o un reflejo cremastérico hiperactivo podrían favorecer esta lesión

La exploración clínica es diagnóstica, sin embargo puede verse dificultada por la existencia de un hematocele o de una luxación testicular profunda. En este caso, aunque se palpaba el testículo en región inguinal, se realizó una TAC abdómino-pélvico para descartar otras lesiones asociadas, confirmándose la localización inguinal del testículo. En ocasiones puede ser útil realizar una ecografía Doppler para valorar la vascularización del testículo sobre todo en traumatismos antiguos. El tratamiento precoz evita las secuelas del traumatismo. En este caso no realizamos toma de fragmento de teste para biopsia ya que no presentaba lesión significativa a la exploración. En general el pronóstico de la luxación testicular es bueno si se realiza de forma precoz la reposición del testículo, evitando alteraciones futuras tales como, testículo doloroso, atrofia testicular, infertilidad ${ }^{9}$.

El tratamiento de elección es quirúrgico, aunque algunos autores logran la reducción manual al cabo de 4 días tras haber cedido la inflama- 
ción. El uso de fármacos miorrelajantes es útil para reducir la luxación de forma indirecta, aunque son poco efectivos (sólo 9 casos descritos). La reducción espontánea es muy infrecuente.

\section{REFERENCIAS}

1. Pérez-Arbej JA, Rosa Arias J, Aranda Lassa JM, Villaroya Rodriguez S, Gil Fabra J, Romero Aguirre F. Traumatismo testicular presentación de tres casos y revisión de la literatura. Arch Esp Urol 1986;39:398-402.

2. Comesaña E, Alexandro Da Silva E, Pereiro B, Meijide F, Pesqueira D, Zungri E. Dislocación unilateral testicular. Arch. Esp. de Urol 1999;52:278-280.

3. Rodríguez Alonso, D Pérez García A, Ojea Calvo B,. Rodríguez Iglesias A,. Alonso Rodrigo JM. Barros Rodríguez, $\mathrm{J}$ et al. Luxación testicular bilateral. Actas Urol Esp 2000;24 (1):58-60.

4. Granados Lorca EA, Ambrosio Teni. VL. Luxación testicular a propósito de un caso. Actas Urol Esp 2000;24(4):360-361,

5. López Alcina E., Martín JC, Fuster A, Pérez J, Puertas M, Moreno J. Dislocación testicular. Aportación de dos nuevos casos. Actas Urol Esp. 2001;25(4):299-302,
6. Alapont Alacreu JM, Dominguez Hinarejos C, Serrano Durba A, Garcia Ibarra F. Dislocación testicular tras traumatismo: A propósito de un caso. Actas Urol Esp. 2002;26 (10) :804-805

7. Horvarth M, Rakotoalizao-Rabejamina F, Massade E, Treboux M. Luxation testiculare traumatique bilatérale. Ann Chir 1993;47: 71-72.

8. Martin Martinez Jc: Traumatismos escrotales y testiculares. Actualizaciones temáticas en urología. 1999. Madaus. Barcelona

9. Hayami S, Ishigooka M, Suzuki Y, Sasagawa I, Nakada T, Mitobe K. K. Pathological changes of traumatic dislocated testis. Urol Int. 1996;56(2):129-32.

Dr. S. Luján Marco

Servicio de Urología

Hospital Universitario La Fe

Avda. Campanar, 21

46009 Valencia

E-mail: slujanmarco@comv.es

(Trabajo recibido el 8 de septiembre 2005) 